\title{
Comparative Evaluation of Ultrasound Kidney Image Enhancement Techniques
}

\author{
Wan Mahani Hafizah \\ Department of Clinical Science \& Engineering, FKBSK \\ Universiti Teknologi Malaysia \\ 81310 Johor Bahru, Malaysia
}

\author{
Eko Supriyanto \\ Department of Clinical Science \& Engineering, FKBSK \\ Universiti Teknologi Malaysia \\ 81310 Johor Bahru, Malaysia
}

\begin{abstract}
Evaluation have been done to different enhancement techniques applied to ultrasound kidney images to see which enhancement techniques is the most suitable techniques that can be applied to the kidney images before segmenting the edge of the kidney. Five common enhancement techniques have been used including the spatial domain filtering, frequency domain filtering, histogram processing, morphological filtering and wavelet filtering. The techniques applied were assessed by few methods which are the observer sensitivity, measuring the image quality by calculating the MSE and PSNR of the image and applying one of the segmentation techniques to the output images. In conclusion, for ultrasound kidney image, if the whole image were taken into consideration (by measuring MSE and PSNR), morphological filtering seems to be the best option in enhancing the image. If the evaluator is concerning more on the kidney edges, enhancement techniques that should be taken into consideration are median filtering and histogram equalization.
\end{abstract}

\section{General Terms}

Image Processing

\section{Keywords}

Keywords are your own designated keywords which can be used for easy location of the manuscript using any search engines.

\section{INTRODUCTION}

Ultrasound (US) imaging has become one of the most preferred imaging techniques in today's medical practice as it is inexpensive, widely available and comparatively safe to the users as well as the operators. However, the presence of speckle noise in all ultrasound images affects the quality of the images which leads to the difficulties in the interpretation of US images. Speckles tend to mask the visibility of the low contrast lesions and reduce the ability of observers to resolve the actual information [1]. Besides, due to the presence of speckles in ultrasound images, the enhancement of US image is extremely difficult especially in image of liver and kidney whose underlying structures are too small to be resolved by large wavelength [2]. They also complicate further image processing, such as image segmentation and edge detection [3]. So, before making any image analysis, suppressing the speckle noise and enhancing the image without losing valuable image features is a very important step.

There are many previous researches done in comparing different ultrasound speckle suppression and image enhancement techniques for US images. Donoho present a soft-thresholding denoising method where the observed image is decomposed into wavelet domain [4]. Thakur et al, by using Donoho's method, have made a comparative study of various wavelet filters with different thresholding values of US images and observed that such denoising methods are effective in the sense that they preserve the edge details besides suppressing the noise [1]. Some other researches which using the wavelet based-filters, also observed that their methods are effective to suppress the noise and enhance the images at the same time. [5-7]. Besides, there are also other researches which use other enhancement methods. Yang et al used histogram matching for enhancing the ultrasound images and their experiment results show their method can leave speckle unchanged and enhance tissue boundaries [8]. Li et al proposed an adaptive image enhancement method using a dynamic filtering for speckle detection [9].

In the present study, five commonly used and have different fundamental theories of image enhancement techniques have been applied on kidney images. The techniques include the spatial domain filtering, frequency domain filtering, histogram processing, morphological filtering and wavelet filtering. Section 2 describes briefly of the different image enhancement techniques that have been implemented while section 3 presents the results and analysis of the experiments.

\section{MATERIALS AND METHODS}

This experiment consists of few steps which are the collecting of 2D ultrasound kidney images, performing different image enhancements techniques, and assessing the performance of each enhancement techniques.

Ultrasound images of normal kidney were taken from volunteers from Department of Clinical Science and Engineering, Faculty of Health Science and Biomedical Engineering, with the help of medical doctor. All images with $416 \times 416$ pixels in size were acquired using Kontron Medical ultrasound machine with $3.5 \mathrm{MHz}$ curved array transducer. Then, all the images were undergone different enhancement techniques discussed in the next subsection.

\subsection{Enhancement Techniques}

Experiment was performed to evaluate different commonly used enhancement techniques for ultrasound images. For this experiment, since we use ultrasound images of normal kidney, there were no abnormal regions to be enhanced. So, the 
enhancement techniques performed were focusing on enhancing the edge of the kidney image.

Few enhancement techniques were proposed and based on the type of image processing used, the techniques can be classified as spatial domain filtering, frequency domain filtering, histogram equalization, morphological processing as well as wavelet filtering. Some of these techniques were commonly used for image enhancement purpose but some were not. Nevertheless, it is important to compare all of these techniques with each other because each of them represents fundamentally different image processing steps. Below is brief discussion on five enhancement techniques mention earlier:

\subsubsection{Nonlinear Spatial Domain Filtering}

One of the most commonly used nonlinear spatial domain filter is median filter. The median filter considers each pixel in the image in turn and looks at its nearby neighbors to decide whether or not it is representative of its surroundings. The median is calculated by sorting all the pixel values from the surrounding neighborhood into numerical order and then replacing the pixel being considered with the middle pixel value. Median filtering is comparatively better that mean filter since it preserves some useful details in an image. It helps in reducing mainly speckle and salt and pepper noise. Median filtering is also called rank filtering [10].

Figure 1 illustrates an example calculation of median value.

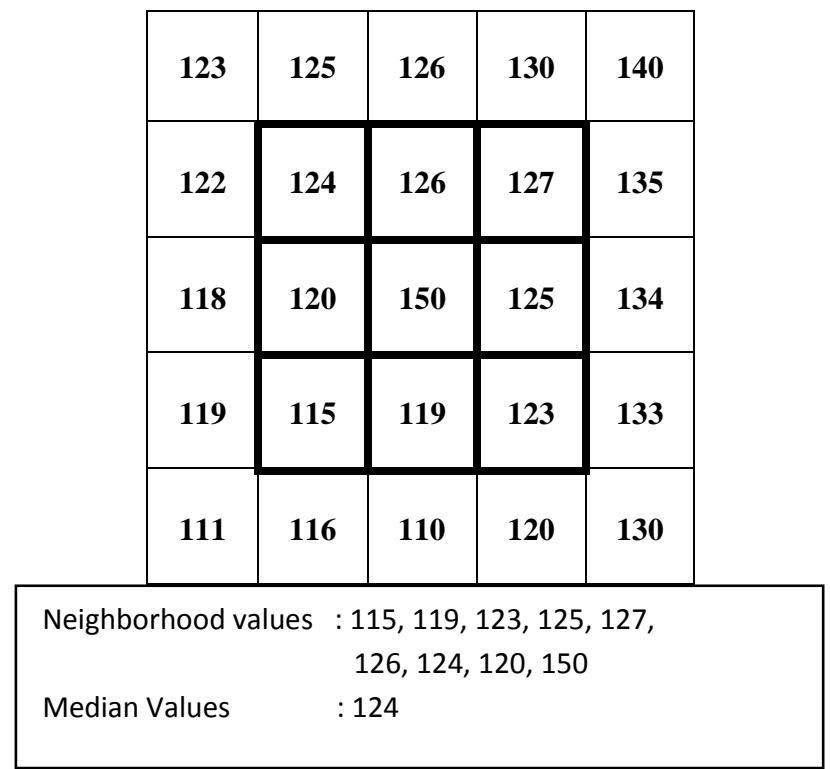

Figure 1: Example for calculating the median value of a pixel neighborhood

\subsubsection{Frequency Domain Filtering}

There are few basic steps in frequency domain filtering. Figure 2 shows the block diagram of the filtering steps in frequency domain. The preprocessing stage might encompass procedures such as determining image size, obtaining the padding parameters and generating the filter. Post processing entails computing the real part of the result, cropping the image and converting it to certain class for storage.

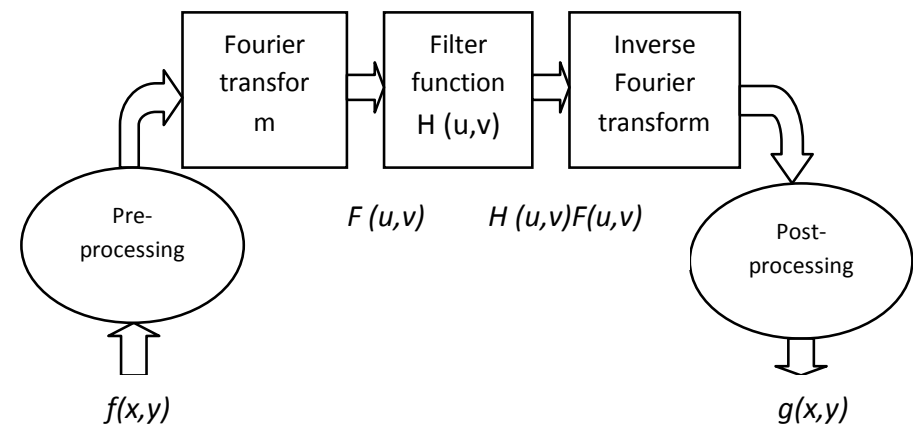

Input image

Filtered image

In frequency domain, the commonly used filter is the low-pass filter based on Gaussian function, since both the forward and the inverse Fourier transforms of a Gaussian are the real Gaussian functions.

The transfer function of a Gaussian low-pass filter (GLPF) is given by

$$
H(u, v)=e^{-D^{2}(u, v) / 2 \sigma^{2}}
$$

where $\sigma$ is the standard deviation and $\mathrm{D}(\mathrm{u}, \mathrm{v})$ is the distance from the origin of the Fourier transform [2].

\subsubsection{Histogram Equalization}

Histogram equalization is an image processing used to improve the visual appearance of an image by adjusting the image histogram. Peaks in the image histogram (indicating commonly used grey levels) are widened, while the valleys are compressed $[11,12]$.

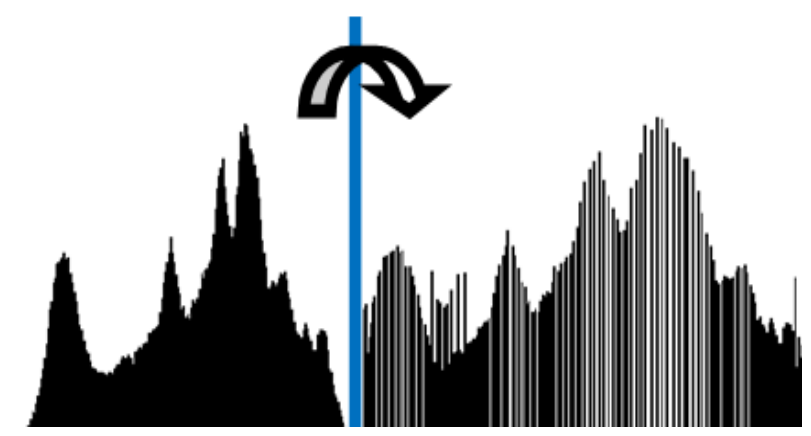

Figure 3: Adjustment of a histogram to distribute intensities

\subsubsection{Morphological Processing}

Morphology is a broad set of image processing operations that process images based on shapes. Morphological operations apply a structuring element to an input image, creating an output image of the same size. In a morphological operation, the value of each pixel in the output image is based on a comparison of the corresponding pixel in the input image with its neighbors. By choosing the size and shape of the neighborhood, you can construct a morphological operation that is sensitive to specific shapes in the input image. 
The most basic morphological operations are dilation and erosion. Dilation adds pixels to the boundaries of objects in an image, while erosion removes pixels on object boundaries. The number of pixels added or removed from the objects in an image depends on the size and shape of the structuring element used to process the image. In the morphological dilation and erosion operations, the state of any given pixel in the output image is determined by applying a rule to the corresponding pixel and its neighbors in the input image. The rule used to process the pixels defines the operation as dilation or erosion [13].

\subsubsection{Wavelets Filtering}

When digital images are to be viewed or processed at multiple resolutions, the discrete wavelet transform (DWT) is the mathematical tool of choice. The most popular technique in wavelet-based filtering is described by Donoho [4].

In wavelet-based filtering, the basic steps for removing the noise are [1]:

1) Decomposing the original image data into l-level of wavelet transform.

2) Performing thresholding of the resultant wavelet coefficients for noise suppression.

3) Performing wavelet reconstruction technique based on the original approximation coefficients.

\subsection{Assessment of Enhancement Techniques}

The assessment between the techniques were made by few methods, consist of:

1. Observer sensitivity: Comparing the output images visually.

2. Image quality measurement: Measuring the output images quality by the traditional distortion measurements such as MSE and PSNR.

3. Image segmentation testing: Applying one segmentation methods to the output images.

During a kidney screening using ultrasound, medical doctors usually measure the kidney length and width. In order to have a more accurate measurement, clear edges of the kidney image is required. Therefore, this experiment will visually compare the output images of different enhancement techniques according to the medical doctors' preference. Besides, the quality of the images will also be measured by the traditional distortion measurements such as MSE and PSNR between the original images and the output images. The mean-squared error (MSE) of the output image is defined as

$M S E=\frac{\sum_{i=1}^{M} \sum_{j=1}^{N}|x(i, j)-\hat{x}(i, j)|^{2}}{M N}$

where $x(i, j)$ is the original image, $\hat{x}(i, j)$ is the output image, and $\mathrm{MN}$ is the size of the image.

The peak signal-to-noise ratio (PSNR) is defined as

$P S N R=20 \log _{10}\left[\frac{\left(2^{n}-1\right)}{\sqrt{M S E}}\right][\mathrm{dB}]$

where $n$ is the number of bits used in representing the pixel of the image. For grayscale image, $n$ is 8 .
Enhancing the edge for edge detection purpose is also important for segmenting the kidney image from its environment. Therefore, we also applied level sets segmentation method to the output image to compare which enhancement techniques enhanced the edge better and can be applied before segmenting the image.

\subsubsection{Level Sets Segmentation}

The level set method was initially proposed to track moving interfaces by Osher et al and has spread across various imaging domains in the late nineties [14].

Generally, level set segmentation is a method for tracking the evolution of contours and surfaces. The image is first smoothed with a Gaussian filter to remove noise, and then the Canny edge detection technique is used to define edges in the image. In level sets segmentation, the user will specify an initial guess for the contour, which is then moved by image driven forces to the boundaries of the desired objects. In such models, two types of forces are considered, the internal and external forces. Internal forces, defined within the curve, are designed to keep the model smooth during the deformation process, while the external forces, which are computed from the underlying image data, are defined to move the model toward an object boundary or other desired features within the image.

For image segmentation process, level sets methods has been chosen. $\mathrm{Li}$ et al. for example proposed a new variational formulation of level set without re-initialization method. This method gives a fast curve evolution and it can be simply implemented via simple finite difference [15].

\section{RESULTS AND ANALYSIS}

The results of the experiment were divided into three parts which are the observer sensitivity, image quality measurements of MSE and PSNR as well as the image segmentation testing.

\subsection{Observer Sensitivity}

After being enhanced by different enhancement techniques, the output images were then showed to the medical doctor to get the opinion scores. Figure 4 shows the sample of ultrasound kidney image which has undergone different image enhancement techniques.

Firstly, the observers were given sets of output images randomly without any mark to show which enhancement techniques being used to each image. They were given option to choose which image is better in defining kidney edges without taking into consideration on any other part being smoothed, blurred, enhanced or even removed away.

According to observers, histogram equalization (Figure 4(b)) is the most preferred enhancement technique followed by wavelet filtering (Figure 4(f)), median filtering (Figure 4(c)), morphological filtering (Figure 4(d)) and Gaussian low-pass frequency domain filtering technique (Figure 4(e)). Histogram equalization technique increase the intensity of the image and the kidney edge is clearer compared to the original image. Median filtering technique smoothes the image but does not enhance the edge whereas Gaussian low-pass frequency domain filtering technique smoothes the image but at the same time the image become blurred. 

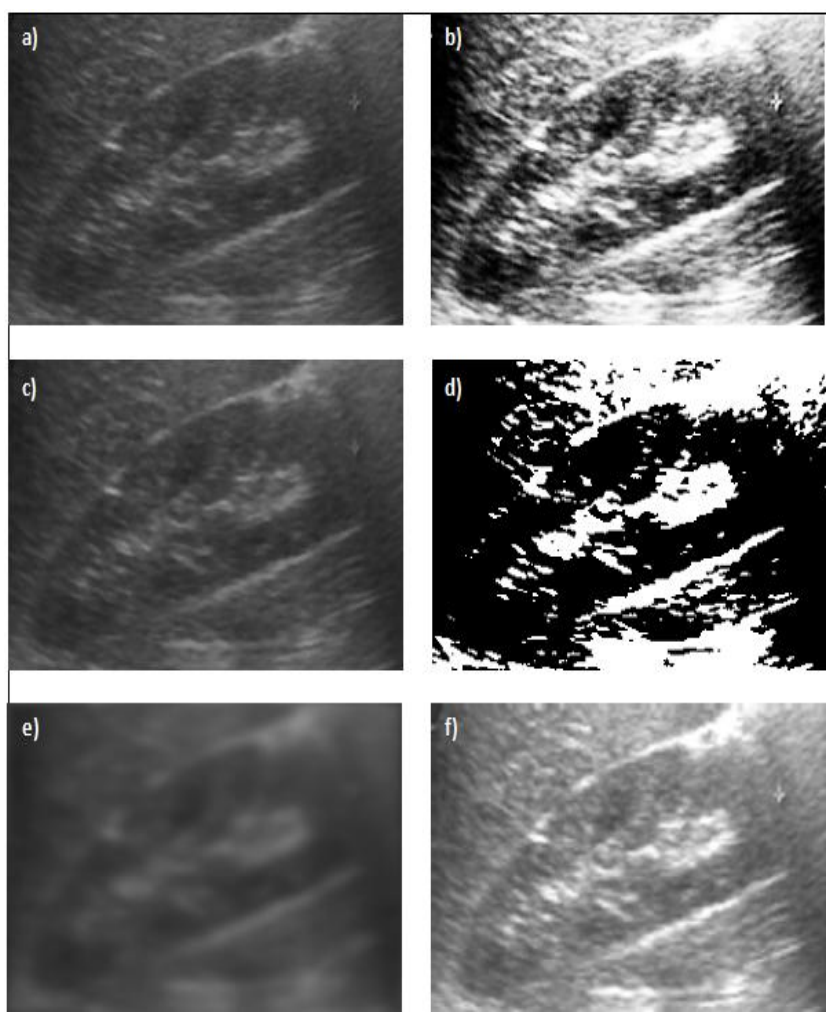

Figure 4: Ultrasound kidney image: a)original image, b)enhanced using histogram equalization, c) nonlinear spatial domain filtering (median filter), d) morphological processing, e) Gaussian low-pass frequency domain filtering, f) wavelet filtering

\subsection{Image Quality Measurements}

The output image quality was also measured by using the meansquared error (MSE) and power signal-to-noise ratio (PSNR) formulas discussed earlier. Table 1 shows the result of MSE and PSNR for different image enhancement techniques.

Based on Table 1, morphological filtering has the highest PSNR value $(66.6566 \mathrm{~dB})$ followed by median filtering $(65.561 \mathrm{~dB})$, Gaussian low-pass frequency domain filtering (54.2179 dB), wavelet filtering $(35.0292 \mathrm{~dB})$ and lastly histogram equalization (34.0544 dB). Higher PSNR means that the image contains more valuable signal compared to the noise in the image. Based on the value of PSNR, it shows that morphological filtering eliminates more noise compared to other enhancement techniques.

Ord filter or order-statistic filter is based on ordering the pixel contained in an image neighborhood and replacing the value of center pixel with the value determined by the ranking result. The best-known order filter is median filter and based on the result in the table, it show that median filter gives higher PSNR compared to other order-statistic filter.
Table 1: MSE and PSNR of different enhancement techniques for ultrasound kidney image

\begin{tabular}{|l|c|c|}
\hline \multicolumn{1}{|c|}{ Enhancement Techniques } & MSE & PSNR[dB] \\
\hline $\begin{array}{c}\text { Nonlinear Spatial Domain } \\
\text { Filtering }\end{array}$ & & \\
$\bullet \quad$ Ord filter & 0.0033 & 48.8461 \\
$\bullet \quad$ Median filter & 0.00007 & 65.561 \\
\hline Gaussian Frequency Domain & & \\
Low-pass Filtering & 0.00097 & 54.2179 \\
\hline Histogram Equalization $\quad$ CLAHE & 0.0235 & 40.3486 \\
$\bullet \quad$ histeq & 0.1003 & 34.0544 \\
\hline Morphological Processing & 0.00006 & 66.6566 \\
\hline Wavelet Filtering & 0.0801 & 35.0292 \\
\hline
\end{tabular}

\subsection{Image Segmentation Testing}

Apart from being assessed visually according to medical doctors' preference and measuring the quality of the image, the output images were also undergone a simple segmentation method in order to automatically test and detect the edge on the enhanced images.

For this level sets method, the user need to set the initial contour first. Number of iterations used in this experiment is 60 . Figure 5 shows the result of segmentation using level sets technique. Since this experiment was focusing on the edge enhancement, an analysis has been done to the image to see the effect of various enhancement techniques to the segmentation process.

Figure 5(a) is the segmentation result of original image before enhancement. Based on the result, it shows that segmentation test result is the best if the images being enhanced using histogram equalization (Figure 5(b)). Other results show that enhancement using wavelet filtering (Figure 5(f)) and median filtering (Figure 5(c)) also give a good segmentation result. However, level sets segmentation methods did not show a good result if using Gaussian low-pass frequency domain filtering for enhancement as this enhancement technique blurred the edge of the image (Figure 5(e)). Thus, the initial contour set will just merge out from the edge of the kidney image. For morphological filtering (Figure 5(d)), the result of segmentation test is also not good as some of the edge of the kidney images has been removed. Therefore, before the segmentation process, enhancement of the image using histogram equalization, wavelet filtering and median filtering can be performed for a better segmentation result. 

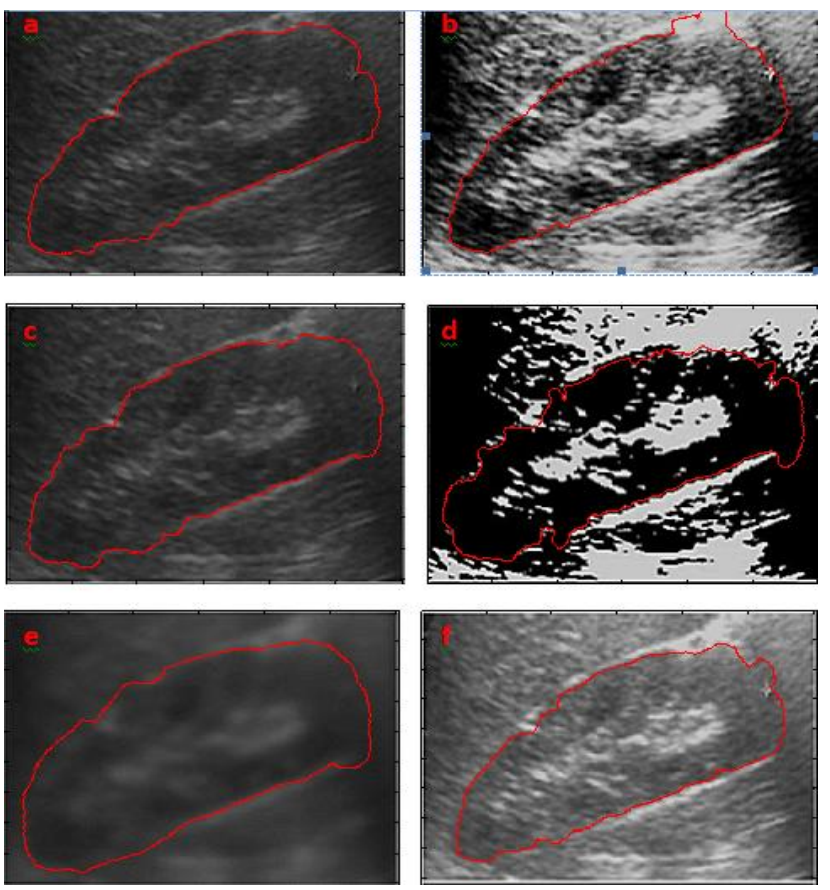

Figure 5: Segmentation image: a)original image, b)enhanced using histogram equalization, c) nonlinear spatial domain filtering (median filter), d) morphological processing, e) Gaussian low-pass frequency domain filtering, f) wavelet filtering

\section{CONCLUSION}

Based on the result, by measuring the MSE and PSNR, it shows that morphological filtering is the best technique in enhancing the ultrasound kidney image compared to other four techniques and median filtering is the second best techniques. On the other hand, based on the observer sensitivity in detecting kidney edges, histogram equalization is more preferred compared to morphological and median filtering. By applying level sets segmentation methods to the output images, it shows that the detection of the edge is better by using histogram equalization, wavelet filtering and median filtering. In conclusion, the performance of five image enhancement techniques has been compared. For ultrasound kidney image, if the whole image were taken into consideration (by measuring MSE and PSNR), morphological filtering seems to be the best option. If the evaluator is concerning more on the kidney edges, median filtering and histogram equalization should be taken into consideration. Developing a new method based on the combination of these two techniques can be the focus of the future research.

\section{ACKNOWLEDGMENTS}

The work is financed by Zamalah Scholarship provided by Universiti Teknologi Malaysia and the Ministry of Higher Education of Malaysia.

\section{REFERENCES}

[1] Thakur, A., Anand, R. S. 2005. Image quality based comparative evaluation of wavelet filters in ultrasound speckle reduction. Digital Signal Processing 15, pp. 455465 .

[2] Shrimali, V., Anand, R. S., Kumar, V. 2010. Comparing the performance of ultrasonic liver image enhancement techniques: a preference study. IETE Journal of Research, Vol 56, Issue 1.

[3] Yu, Y., Acton, S. T.2002. Speckle reducing anistrophic diffusion. IEEE Trans on Imag Process, Vol 11, pp 12601270 .

[4] Donoho, D. L., 1995. Denoising by soft-thresholding. IEEE Trans. Inform. Theory 41, pp 613-627.

[5] Yue, Y., Croitoru, M. M., Bidani, A., Zwischenberger, J. B., Clark, J. W. 2005. Ultrasound speckle suppression and edge enhancement using multiscale nonlinear wavelet diffusion. Proceeding of IEEE Engineering in Medicine and Biology $27^{\text {th }}$ Annual Conference.

[6] Rallabandi, V. P. S. 2008. Enhancement of ultrasound images using stochastic resonance-based wavelet transform. Computerized Medical Imaging and Graphics 32, 316-320.

[7] Sudha, S., Suresh, G. R., Sukanesh, R. 2009. Speckle noise reduction in ultrasound images by wavelet thresholding based on weighted variance. International Journal of Computer Theory and Engineering, Vol 1, No 1.

[8] Yang, X., Zhang, J., Peng, B., You, S. 2010. An adaptive edge enhancement method based on histogram matching for ultrasound images. IEEE International Conference on Computational and Information Sciences.

[9] Li, X., Liu, D. C. 2007. Ultrasound image enhancement using dynamic filtering. IEEE Fourth International Conference on Image and Graphics.

[10] Arulmozhi, K., Perumal, S. A., Kannan, K., Bharati, S. 2010. Contrast improvement of radiographic images in spatial domain by edge preserving filters. IJCSNS International Journal of Computer Science and Network Security, VOL.10 No.2.

[11] Cheng, H. D., Shi, X. J. 2004. A simple and effective histogram equalization approach to image enhancement. Digital Signal Processing 14, 158-170.

[12] Zhu, H., Chan, F. H. Y., Lam, F. K. 1999. Image contrast enhancement by constrained local histogram Equalization. computer vision and image understanding, Vol. 73, No. 2, pp. 281-290.

[13] Matsopoulos, G. K., Marshall, S. 1994. Use of morphological image processing techniques for the measurement of a fetal head from ultrasound images. Pattern Recognition, Vol. 27, No. 10, pp. 13171324.

[14] Osher, S., Sethian, J. A. 1988. Fronts propagating with curvature dependent speed: Algorithms based on HamiltonJacobi Formulations. Journal of Computational Physics, volume 79, pp. 12-49.

[15] Li, C., Xu, C., Gui, C., Fox, D.M. 2005. Level set evolution without re-initialization: a new variational formulation, in IEEE Computer Soceity Conference on Computer Vision and Pattern Recognition(CVPR). 